\title{
Transmission Electron Microscopic Studies on the Skin of Goat (Capra hircus)
}

\author{
U.P. Mainde ${ }^{1}$, S.B. Banubakode ${ }^{1}$, N.C. Nandeshwar ${ }^{1}$, R.Y. Charjan ${ }^{1}$, S. Sathapathy ${ }^{2}$ and A.M. Salankar $^{1}$ \\ ${ }^{1}$ Department of Veterinary Anatomy and Histology, Nagpur Veterinary College, MAFSU, Nagpur, Maharashtra, INDIA \\ ${ }^{2}$ Department of Anatomy and Histology, CVSc. and A.H., OUAT, Bhubaneswar, Odisha, INDIA \\ "Corresponding author: UP Mainde; E-mail: drumeshmainde@gmail.com
}

Received: 04 July, 2020

Revised: 15 Aug., 2020

Accepted: 29 Aug., 2020

\begin{abstract}
The present study was conducted on skin of goats of either sex to study the age wise changes in the ultrastructure of different components of skin. It was observed that the epidermis consisted of keratinocytes and non-keratinocytes. The stratum corneum was a multicellular structure in lateral neck region. The cell membrane was serrated and arranged in an over lapping manner giving compactness to the skin. The cells of stratum granulosum had oval to oblong nuclei. The cytoplasm showed ample amount of keratohyaline granules mostly located in the peripheral zone. The cells of stratum spinosum had oval nuclei with numerous secretory granules in the cytoplasm. The skin at ventral thoracic region showed presence of melanocytes in the cells of stratum basale. The plasma membrane of stratum basale cells showed gap junction, tight junction and intermediate junction. Electron microscopically, the cells of stratum spinosum in ventral neck region showed indented nuclei with one broad pole and another narrow pole. The special cells such as Merkel cells and melanocytes were located in the deep epidermal or epidermodermal junction. The papillary layer of dermis of dorsal neck region during the present study showed circular axons. The acini of the sebaceous gland were composed of large central cells and peripheral small cells, surrounded by connective tissue. The sweat glands were composed of columnar cells with elongated nuclei and euchromatin. The present study would form a baseline data on the transmission electron microscopic study of skin of goat which would be helpful in future research prospective.
\end{abstract}

\section{HIGHLIGHTS}

(0 The cytoplasm of stratum granulosum contained ample amount of keratohyaline granules mostly located in the peripheral zone.

0 The plasma membrane of stratum basale cells showed gap junction, tight junction and intermediate junction.

0 Merkel cells and melanocytes were located in the deep epidermal or epidermo-dermal junction.

Keywords: Goat, skin, transmission electron microscopy

The skin is the largest organ in mammalian body and constitutes 8.5 per cent of live body weight (Mainde et al., 2019a). The skin of goat is often used for making gloves and boots. It is a versatile organ, which is extremely important for protection, perception, water regulation and wound healing (Razvi et al., 2015; Mainde et al., 2018 and Mainde et al., 2019b). The skin of goat is considered extremely durable used by the tannery Industry to make rugs and carpet binding. Skin is thicker on the dorsal and extensor surfaces than on the ventral and flexor surfaces. It is thicker in the male. The capacity of the skin to move and be stretched depends on its own thickness, the number of its folds, its intrinsic elasticity and firmness of fixation by the tela subcutanea and age of individual (Mainde et al., 2019c). The thickness of skin varies on the basis of species, breed, age, sex and body region in domestic animals (Mainde et al., 2019c). The literature on the ultrastructure of skin of goat is scanty and therefore, the present study was undertaken to develop a baseline data for future research perspectives.

How to cite this article: Mainde, U.P., Banubakode, S.B., Nandeshwar, N.C., Charjan, R.Y., Sathapathy, S. and Salankar, A.M. (2020). Transmission electron microscopic studies on the skin of goat (Capra hircus). J. Anim. Res., 10(5): 711-716.

Source of Support: None; Conflict of Interest: None क क 


\section{MATERIALS AND METHODS}

The skin samples of goats were collected from healthy animals irrespective of sex from slaughter house of Nagpur Municipal Corporation and also from animals after natural death. Processing and preparing samples for transmission electron microscopic (TEM) study (Bozzola and Russell, 1999). For ultrastructural studies, the tissue samples were primarily fixed in 3\% glutaraldehyde in 0.05 $\mathrm{M}$ phosphate buffer (pH7.2) in vials for 24 hours at $4-10^{\circ} \mathrm{C}$ and washed with three changes of $0.05 \mathrm{M}$ phosphate buffer (pH7.2) at $4-10^{\circ} \mathrm{C}$ for 2 hours in each change. Secondary fixation was done with $2 \%$ aqueous osmium tetraoxide in the same buffer for 2 hours at $4-10^{\circ} \mathrm{C}$. Dehydration was achieved by immersing the tissue in ascending grades of alcohol sequentially in $30 \%, 50 \%, 70 \%, 90 \%$ (two changes) and absolute alcohol (two changes in each) for 15 minutes in each at $4-10^{\circ} \mathrm{C}$. Clearing was done with three times infiltrations in transitional solvent propylene oxide for 15 minutes in each. Infiltration was achieved by passing the tissue with propylene oxide and resin mixture for overnight at $35^{\circ} \mathrm{C}$. After infiltration, tissue samples were embedded in pure resin mixture and kept at $45^{\circ} \mathrm{c}$ for 24 hours. The final step in embedding was curing. For curing, the tissue blocks were kept at $60^{\circ} \mathrm{C}$ for 24 hours for polymerization of resin mixture. With Leica Ultra cut UCT-GA-D/E ultramicrotome, with a glass knife, semithin sections of 1-2 $\mu \mathrm{m}$ were cut and transferred into tiny droplets of distilled water then on acid cleaned glass slide and kept on a hot plate $\left(70-90^{\circ} \mathrm{C}\right)$ until the water was evaporated. After the slide had cooled, a drop of staining solution ( $1 \%$ toluidine blue dissolved in $1 \%$ aqueous sodium borate solution) was placed over the dried sections and reheated on hot plate until the stain was dried. By light microscopic examination, (Olympus AX-70) the desired skin area was identified in section, which needed to be seen with a transmission electron microscope. After adjusting the thickness control to an approximate setting on ultramicrotome, the ultrathin sections of 900$1000 \mathrm{~A}^{\mathrm{o}}$ of golden color were cut. The ultrathin sections were mounted on the clean copper grid with 200 mesh. After drying, the grid was immediately kept in grid holder. The sections were stained with saturated aqueous uranyl acetate. After staining, grid was gently rinsed in distilled water and counter stained with 490 lead citrate. For ultrastructural observations, the grid was placed in the specimen holder and introduced into the specimen chamber of the transmission electron microscope (Model - Hitachi, H-7500). The electron microscopic work was done at Ruska Laboratory, College of Veterinary Science, Rajendra Nagar Hyderabad, India. Electron microscopic photographs at various magnifications were taken for depiction of ultrastructure of various parts of the skin at different age groups.

\section{RESULTS AND DISCUSSION}

The electron microscopic study of goat skin revealed region specific differences in terms of cellular morphology and degree of keratinization amongst neck, thorax, abdomen and thigh regions. The variations were chiefly observed in terms of shape of nucleus, chromatin content and cellular junctions, which reflected on the cellular activity and degree of cornification. The cellular activity was found diminished with the advancement of peeling process in the epidermal layer. The special cells such as Merkel cells and melanocytes were located in the deep epidermal or epidermo-dermal junction. The epidermis was consisted of keratinocytes and non-keratinocytes.

In dorsal thorax region, melanocytes were rested on the basal lamina. The cells of basal lamina showed uniform density, elongated nucleus with foamy nucleoplasm. The delicate protoplasmic processes were present on the cells of basal lamina. These processes were found to provide closer links between the dermis and the epidermis. In dorsal abdomen region, the basal cells were tall columnar pyramidal in shape with oval and elongated nucleus. The ventral border of some basal cells showed faint serration. The nucleoplasm of basal cells presented euchromatin and heterochromatin with distinct basal lamina (Fig. 1).

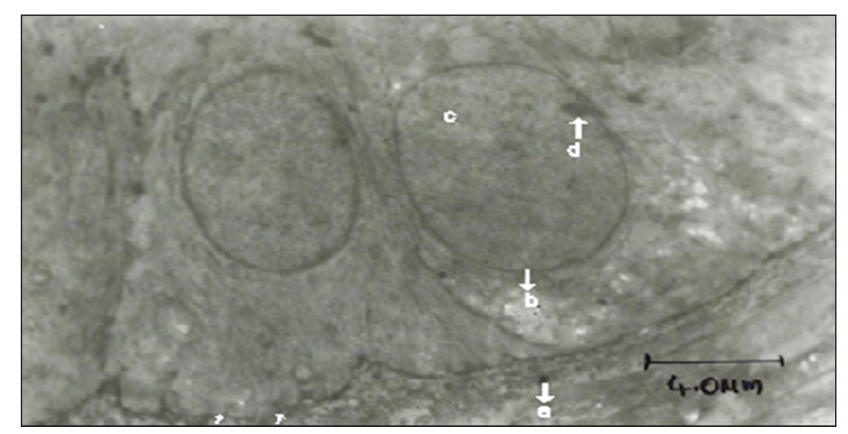

Fig. 1: Transmission electron photograph of skin of goat in dorsal abdomen region showing cells of stratum basale (a) Basal lamina (b) Nucleus (c) Euchromatin (d) Heterochromatin (x 2500) 
The protoplasmic process of basal cells of stratum basale layer extended into the dermis to provided close union between epidermis and dermis. These extensions of protoplasmic processes of ventral border of basal cells of stratum basale into the dermis may be responsible for better anchorage. The presence of tall columnar pyramidal shaped basal cells with oval or elongated nuclei recorded during present study reflects the higher cellular activity. The presence of euchromatin in the cytoplasm of basal cells is indicative of optimum cytocellular activity.

During the present study, it was noted that the melanocytes were cuboidal to columnar type. The nuclei of melanocyte were oval to oblong and with indentations. The heterochromatin was present below the nuclear membrane. The cytoplasm was pale with dark secretory material at periphery. These melanocytes are supposed to be responsible for imparting colour to skin and hair. The plasma membrane of cells of stratum spinosum was serrated and its cytoplasm showed presence of melanosomes (Fig. 2).

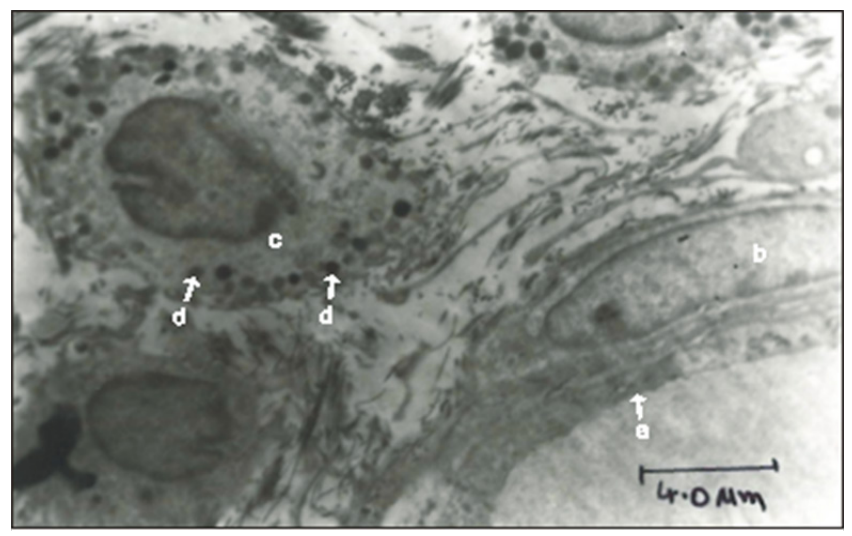

Fig. 2: Transmission electron photograph of skin of goat in dorsal thoracic region showing basal cell resting on the basal lamina (a) Basal lamina (b) Foamy nucleoplasm (c) Melanocytes (d) Melanin granules (x 2500)

The papillary layer of dermis of dorsal neck region during the present study showed circular axons. The axon was surrounded by bi-laminar boundary and a distinct Schwann cells. The axons consisted of nerve fibrils of different size and were surrounded by adipose tissue (Fig. 3). The secretion of myelin was observed in the Schwann cells, which indicated the active stage of axon. Electron dense material was seen located near Schwann cells, which reflects the higher metabolic activity of the cells.

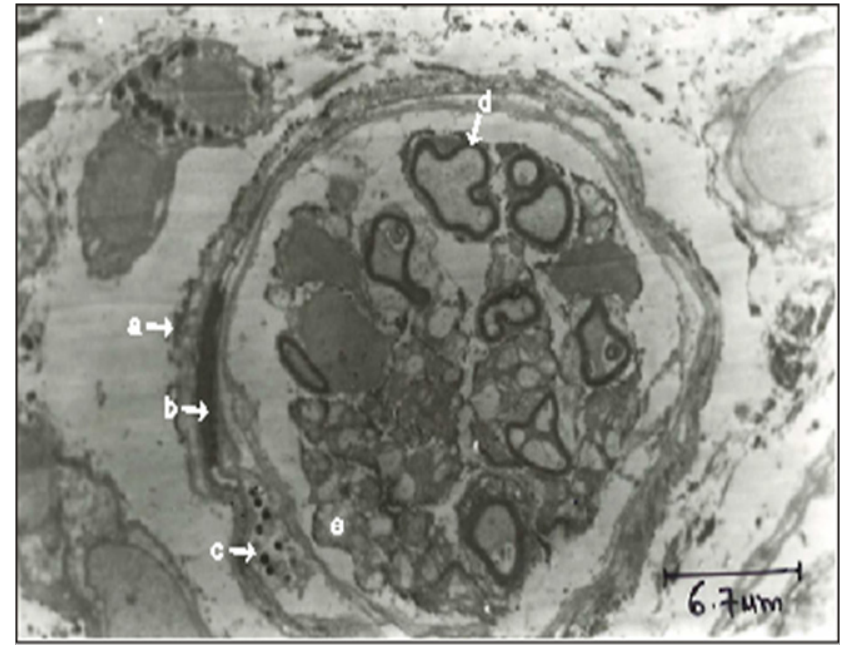

Fig. 3: Transmission electron photograph of skin of goat in dorsal neck region showing axon innervating the Merkel cell (a) Axon (b) Electron dense material of myelin (c) Schwann cell (d) Nerve fibrils (e) Adipocytes (x 1500)

It was noted that, the arrector pili muscle in the dermis was composed of smooth muscle cells. These cells were stellate shaped and had elongated prominent nucleus with scanty peripheral pale cytoplasm. The abundant heterochromatin was distributed on the inner side of the nuclear membrane (Fig. 4). At some places, it was projected into nucleoplasm. The nucleoplasm also showed fair amount of heterochromatin in the form of strands, which is indicative of higher metabolic activity of muscle cells.

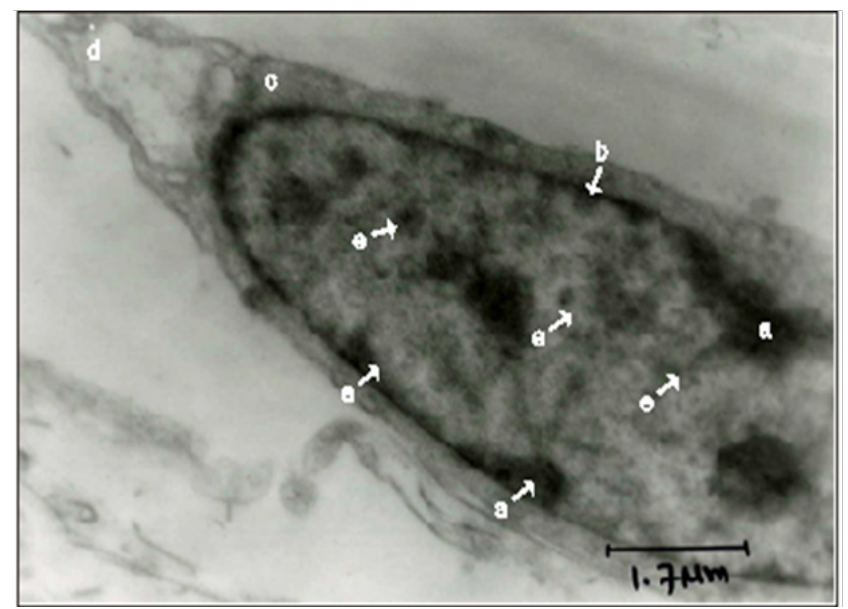

Fig. 4: Transmission electron photograph of skin of goat in dorsal thoracic region showing smooth muscle cells of arrector pili (a) Heterochromatin (b) Nuclear membrane (c) Cytoplasm (d) Tapering end (e) Strands of heterochromatin (x 6000) 
The acini of the sebaceous gland were composed of large central cells and peripheral small cells, surrounded by connective tissue. The nuclei were mostly euchromatic and polymorphic in shape. The cytoplasm showed uniform dense fatty material, which is indicative of secretory activity of the cells. The secretion of the gland keeps the hair shiny.

The present study indicated that the sweat glands were composed of columnar cells with elongated nuclei and euchromatin. The apical part of cell present number of microvilli projected into the lumen (Fig. 5). In agreement with the findings of the present study, Taha and Abdalla (1980) stated that the secretory cells were polyhedral in shape with round or oval basal nucleus in sweat gland of camel. They further reported that the microvilli were present in luminal surface. The presence of columnar cells with elongated nuclei projected microvilli in the lumen of the sweat gland is indicative of activity of sweat gland even in the hairy animals.

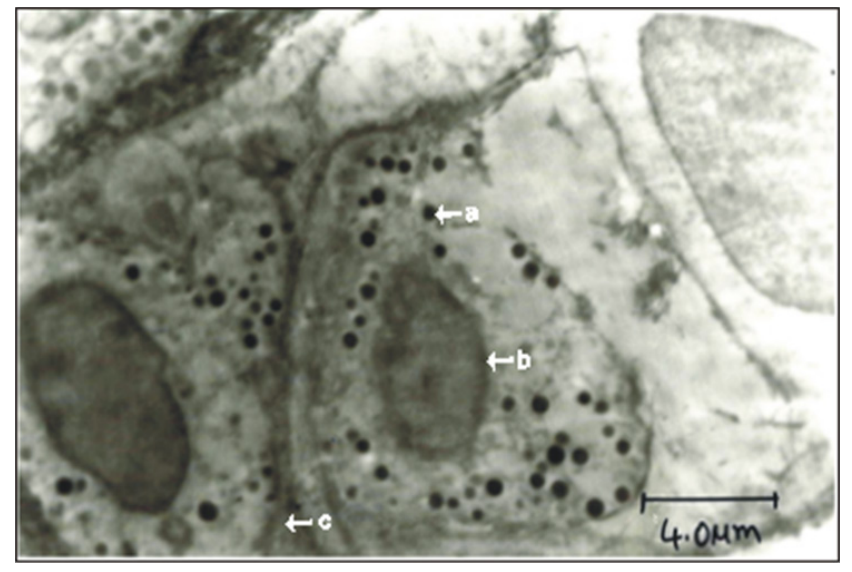

Fig. 5: Transmission electron photograph of skin of goat in lateral abdomen region showing intercellular gap junction between acinar cells of sweat gland (a) Secretory granules (b) Nucleus (c) Intercellular gap junction between acinar cells (x 3000)

In the present study, it was noted that the cortex of the hair follicle was homogenous in nature. The centrally located medulla showed varying electron density. Many irregular shaped air spaces were found in the medulla. This peculiar arrangement is probably species specific in animal hair. However, the literature on this aspect is obscure.

During the present study, it was noted that the cells of stratum basale in the lateral neck region showed distinct boundary, pale cytoplasm and oval nucleus, which was mostly euchromatic. However, it was heterochromatic at the periphery. This type of cytocellular morphology observed during the present study may be indicative of active phase of cells.

The electron microscopic study during present work showed that the cells of stratum granulosum had oval to oblong nuclei. In stratum spinosum nuclear membrane was distinct and nuclear material was uniformly distributed and beads of heterochromatin were seen attached to the internal nuclear membrane. The cytoplasm of stratum granulosum showed ample amount of keratohyaline granules mostly located in the peripheral zone and the nuclei were elongated. The cells of stratum granulosum were well demarcated from stratum spinosum, which had oval nuclei. The cytoplasm contained numerous secretory granules. In agreement with the findings of the present study, Pfeiffer et al. (2006) reported that the cytoplasm was electron dense due to presence of melanin granules. They further reported the presence of heterochromatin towards the periphery of cytoplasm.

The electron microscopic study revealed that the cells of stratum corneum were composed of multicellular structure in lateral neck region. The cell membrane was serrated and arranged in an over lapping manner giving the compactness to the skin. The cells of superficial layer showed more keratinization and the nuclei were degenerated. Similar observations were reported by Pfeiffer et al. (2006) in camel. They stated that the cytoplasm was composed of clearly packed electron lucent spherical and ovoid secretory granules.

The present study revealed that the intercellular gap junctions between acinar cells present in sweat gland. The elongated nucleus was present in the cells of sweat gland. The cytoplasm showed abundant secretory granules. These observations of the present study were contradictory to the findings reported by Mainde et al. (2019c) in goat. They mentioned that the gap junctions were absent. Further, they stated that basal cells were not interconnected by desmosome and their cytoplasm was rich in ribosomes and contained glycogen deposits and filament bundle.

The arrector pili muscle, showed bundle of muscle fibrils surrounded by collagen fibers in its cross section during the present study. Different sizes of muscle fibrils were present in each bundle Electron microscopically. These 
muscle fibrils were electron dense. Similar observations were recorded by Pfeiffer et al. (2006) in camel. They stated that the ultra structure of myocytes of arrector pili muscles showed numerous fusiform densities in its cross section with centrally placed nuclei. They further mentioned that the myocytes were encircled by thick basement membrane and collagen fibers were present in between the cells. This peculiar ultrastructure of arrector pili muscle is probably responsible for bringing out the secretory function of sweat and sebaceous gland.

Electron microscopically, the cells of stratum basale in the ventral neck region were observed as cuboidal to columnar in shape and had clear cell boundaries. The nuclei were oval and indentated. The nucleoplasm had heterochromatin as well as euchromatin. The nuclei of the cells of stratum basale in lateral neck region, however, were oval shaped. Multiple projections were found on the deeper side of the cells. These projections were found to enter the dermal tissue. These findings of the present study coincided with the observations recorded by Mainde et al. (2019c) in goat. They stated that the epidermal basal cells were connected with the dermis by the cytoplasmic filament of the basal cells. The presence of multiple projections on the deeper part of the cells of stratum basale might be responsible for better attachment between dermis and epidermis.

The skin at ventral thoracic region showed presence of melanocytes in the cells of stratum basale. The nucleus was irregular to indentated type and centrally located. Nucleoplasms showed euchromatin and number of vacuoles in the cytoplasm of melanocytes. The cells of stratum basale in ventral abdomen region were columnar type. The nucleus was elongated and had peripheral heterochromatin. The plasma membrane of basale cells showed gap junction, tight junction and intermediate junction (Fig. 6). The nucleus of the cells of stratum basale in ventral abdomen region was very prominent and elongated in shape. The nucleoplasm was euchromatic. The cytoplasm of the cell was homogenous with mitochondria and vacuoles. Similar findings were reported by Samuelson (2007) in domestic animals. He reported that the melanocytes possessed number of arm like processes, which were extended into stratum spinosum. He correlated the presence of these processes for bringing out the movement of melanosomes from stratum basale to stratum spinosum.

Journal of Animal Research: v. 10, n. 5, October 2020

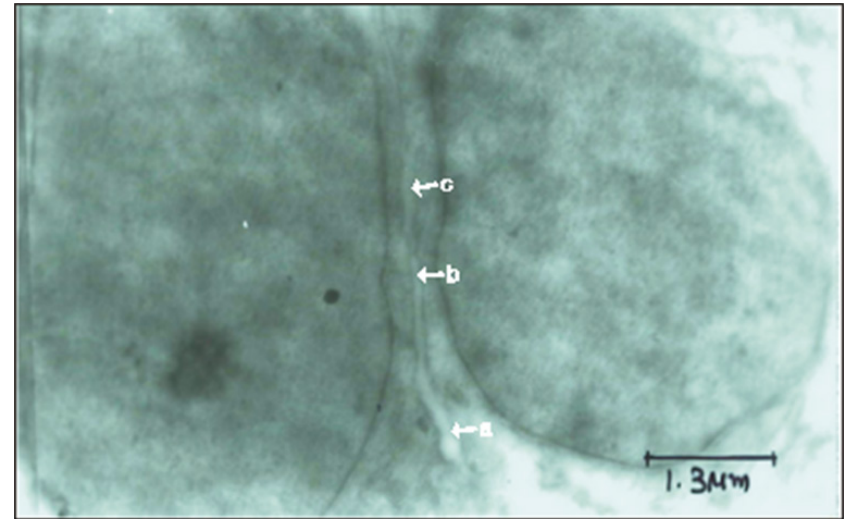

Fig. 6: Transmission electron photograph of skin of goat in ventral abdomen region showing cells of stratum basale (a) Gap junction (b) Tight junction (c) Intermediate junction (x 8000)

Electron microscopically, the cells of stratum spinosum in ventral neck region showed indented nuclei with one broad pole and another narrow pole. The nuclear membrane was very fine with slightly more electron density at periphery. The nucleus was surrounded by lysozomes, mitochondria, golgi bodies and some electron dense keratohyaline granules along with secretory granules in the cytoplasm of the cells (Fig. 7).

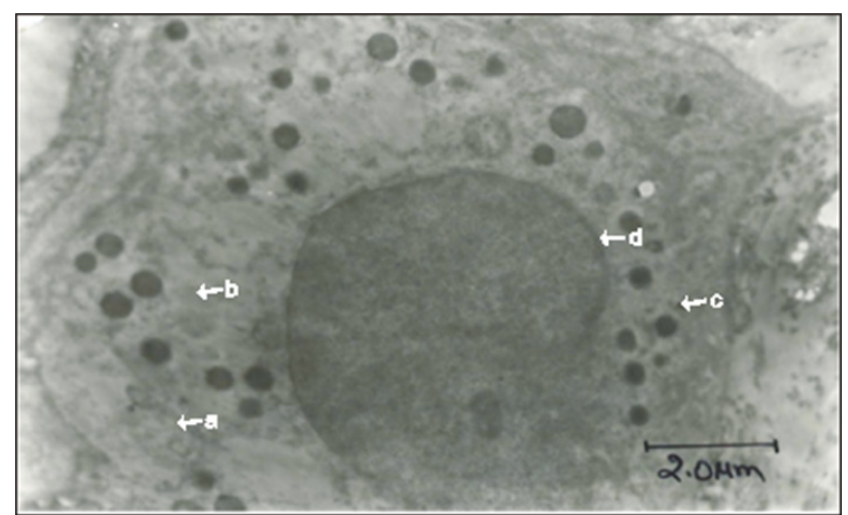

Fig. 7: Transmission electron photograph of skin of goat in ventral neck region showing stratum spinosum (a) Mitochondria (b) Lysosomes (c) Secretory material (d) Electron density at periphery of nucleus (x 5000)

The cells of stratum spinosum in ventral abdominal region were spindle shaped with oval nuclei and peripheral cytoplasm. The cells had tapering ends perpendicular to surface. Cytoplasm showed uniformly distributed keratohyaline granules. Hyper chromitization was seen in nuclear membrane. In agreement with the findings of the 
present study, Pfeiffer et al. (2006) in camel skin reported that the cells of stratum spinosum were composed of large spherical nuclei with sparse chromatin electron lucent cytoplasm and small electron dense granules.

The peripheral flattened secretory cells were found to secrete serous secretion in the lumen of sweat gland. The secretory drops were also seen in the duct. The epithelial cells of the sweat gland were found rested on the basement membrane in the ventral abdomen region. The cytoplasm of glandular epithelium was uniform with foamy appearance containing cell organelles and secretory material.

Intercellular connective tissue was seen in between secretory cells of sweat gland in ventral thoracic region. Central lumen of the acini was surrounded by secretory acinar cells provided with microvilli and had elongated nuclei with uniform cytoplasm. The nucleoplasm showed heterochromatin. The acinar cells showed homogenous cytoplasm containing serous secretion giving pale appearance to the cells. The peripherial nucleoplasm showed heterochromatin granules. Prominent lumen was found at the center of the acini. Similar findings were reported by Pfeiffer et al. (2006) in camel. They stated that the apical part of cells of sweat gland showed closely packed microvilli.

Present study revealed that translucent secretory fatty material was present in sebaceous gland. The bundle of collagen fibers in the papillary and reticular layer of dermis in ventral neck region were found arranged in oblique direction. The peripheral fibers showed low electron density as compared to the central fibers at axis. These observations of the present study coincided with the findings reported by Pfeiffer et al. (2006) in camel. They observed occasional small lipid droplets and other usual organelles in fully differentiated cells of sebaceous gland.

\section{CONCLUSION}

The electron microscopic study of goat skin revealed region specific differences in terms of cellular morphology and degree of keratinization amongst neck, thorax, abdomen and thigh regions. The variations were chiefly observed in terms of shape of nucleus, chromatin content and cellular junctions, which reflected on the cellular activity and degree of cornification. The present study would form a baseline data on the transmission electron microscopic study of skin of goat which would be helpful in future research prospective.

\section{REFERENCES}

Bozzola, J.J. and L.D. Russell. 1999. Electron Microscopy: "Principals and Techniques for Biologists ( $2^{\text {nd }} E d n$.)", Jone Barttett Publishers, Subhure Masschusetts, pp. 19-144.

Mainde, U.P., Banubakode, S.B., Nandeshwar, N.C., Charjan, R.Y., Salankar, A.M. and Patil, D.V. 2018. Histological and histochemical studies of epidermis of goat (Capra hircus). Indian J. Vet. Anat., 30(2): 116-117.

Mainde, U.P., Banubakode, S.B., Nandeshwar, N.C., Charjan, R.Y. and Salankar, A.M. 2019a. Histological and histochemical studies of sebaceous glands of goat (Capra hircus). Indian J. Vet. Anat., 31(1): 154-155.

Mainde, U.P., Banubakode, S.B., Nandeshwar, N.C., Bonde, S.W., Charjan, R.Y., Salankar, A.M. and Gedam, P.M. 2019 b. Histological and histochemical studies of sweat glands of goat (Capra hircus). Int. J. Sci. Env. Tech., 8(1): 63-69.

Mainde, U.P. 2019c. Histological, histochemical and ultrastructural studies on the skin of goat (Capra hircus). MVSc. thesis submitted to Maharashtra Animal and Fishery Sciences University, Nagpur, Maharashtra.

Pfeiffer, C. J. 2006. Ultrastructural analysis of the integument of a desert adapted mammal, the one humped Camel (Camelus dromedarius). Anat. Histol. Embryol., 35: 97-103.

Razvi, R. 2015. Skin and its ageing. The NE Vet., 14: 16-18.

Samuelson, D.A. 2007. Textbook of Veterinary Histology, Saunders Elsevier, pp. 271-299.

Taha, A.A. and Abdalla, A.B. 1980. Light and electron microscopy of the sweat glands of the dromedary Camel. Acta Vet. Brno., 49: 31-35. 